\title{
Carrières de licencié.e.s.
}

Revenir sur le passé pour saisir les destins des chômeurs de Moulinex

Redundant employees' careers. Looking at the professional past to understand what became of Moulinex's redundant workers

\section{Manuella Roupnel-Fuentes}

\section{(2) OpenEdition}

1 Journals

\section{Édition électronique}

URL : http://journals.openedition.org/travailemploi/6325

DOI : 10.4000/travailemploi.6325

ISSN : 1775-416X

Éditeur

DARES - Ministère du Travail

\section{Édition imprimée}

Date de publication : 1 avril 2014

Pagination : 19-36

ISSN : 0224-4365

\section{Référence électronique}

Manuella Roupnel-Fuentes, "Carrières de licencié.e.s. ", Travail et Emploi [En ligne], 138 | avril-juin

2014, mis en ligne le 01 avril 2016, consulté le 30 avril 2019. URL : http://journals.openedition.org/ travailemploi/6325 ; DOI : 10.4000/travailemploi.6325 


\title{
Carrières de licencié.e.s. Revenir sur le passé pour saisir les destins des chômeurs de Moulinex
}

\author{
Manuella Roupnel-Fuentes $\left.{ }^{*}\right)$
}

\begin{abstract}
Que sont devenu.e.s les 3000 Moulinex des cinq usines de Basse-Normandie licencié.e.s en septembre 2001 ? Pour répondre à cette question, nous nous appuyons sur les résultats d'une enquête menée par questionnaires auprès de 830 ancien.ne.s salarié.e.s au cours de l'automne 2003 et par entretiens approfondis. Le traitement statistique montre que la structuration de la main-d'œuvre au sein de Moulinex, fortement segmentée selon le sexe, l'âge et la qualification, se retrouve transposée dans les trajectoires ultérieures sur le marché du travail. La mise en regard du passé professionnel avec les situations retrouvées deux ans après la fin de l'emploi fait émerger trois figures typiques de " carrières» de licencié.e.s, allant du rebond difficile des ouvrières non qualifiées à des poursuites de carrière des techniciens diplômés. Cette mise au chômage, massive et localisée dans le cadre d'un large plan de licenciement, prouve aussi la forte influence de la situation géographique et, dans une moindre mesure, de la formation professionnelle. Le rôle du diplôme initial apparaît ambigu: s'il peut permettre aux plus diplômés d'échapper au chômage, de quitter l'entreprise avant que ne survienne le plan social ou de bénéficier plus facilement d'une mesure d'âge, c'est une ressource visiblement moins déterminante pour ceux qui se retrouvent au chômage.
\end{abstract}

Les suppressions d'emplois de vaste ampleur jalonnent l'histoire industrielle et prennent des formes très variées : elles peuvent être limitées ou représenter un grand nombre de licenciements, faire l'objet d'une programmation et d'une concertation entre les partenaires concernés ou, au contraire, intervenir de manière subite et inattendue, être organisées en interne ou conduire à la disparition de l'entreprise suite à une liquidation judiciaire, etc. Tel fut le cas de la fermeture de Moulinex, à l'issue de la liquidation judiciaire du groupe MoulinexBrandt prononcée en octobre 2001 par le tribunal de commerce de Nanterre (le siège étant à la Défense) avec pour conséquence le licenciement de plus de 4500 personnes en France. Le plan de reprise par Seb épargne trois usines ${ }^{(1)}$, mais entraîne le licenciement de près de 3000 salarié.e.s (sans compter les emplois menacés chez les sous-traitants, estimés à environ 1000 au moment du dépôt de bilan). La faillite de l'entreprise empêche toute possibilité de reclassement «en interne» des salariés( ${ }^{(2)}$. Les dispositifs prévus pour favoriser leur reclassement «en externe» visent alors à aider leur réintégration sur le marché du travail (congé ou convention de

(*) ESO-Angers (Espaces et sociétés) - UMR 6590, IUT d'Angers-Cholet; roupnel@univ-angers.fr

(1) Ces sites sont Fresnay-sur-Sarthe (Sarthe), Villaine-laJuhel et Mayenne (Mayenne) ainsi qu'une partie de l'usine de Saint-Lô (Manche).

(2) Même si, le 6 août 2010, la justice a déclaré leur licenciement en 2001 "sans cause réelle ni sérieuse» et alloué à 190 anciens salariés des indemnités allant de 1500 à 50000 euros. conversion ${ }^{(3)}$ ) ou leur retrait de celui-ci au travers de «mesures d'âge» (préretraite allocation spécifique - Fonds national pour l'emploi [AS-FNE], préretraite amiante et allocation équivalent retraite).

Trois mois après la mise en faillite de Moulinex, et pour une durée initiale de douze à dix-huit mois, le plan social prévoit la mise en place d'une cellule emploi sur chacun des cinq sites fermés afin de permettre un accompagnement des salarié.e.s dans leur démarche de recherche de solutions, qu'elles soient d'emploi ou sociale. Face au plus grand plan de licenciements depuis la catastrophe du Creusot (Outin, Perriet-Cornet, 1991), la tâche des consultants de ces cellules a d'abord été d'accueillir tous.tes les salarié.e.s et leur colère puis, avec le temps, de définir les réponses les plus adaptées aux problématiques de chacun. L'image souvent dépeinte par les médias des «Moulinex» qui se sont mobilisés contre cette fermeture est celle d'une population homogène, essentiellement des ouvrières âgées et peu qualifiées. Or, ce portraitrobot sous-estime la diversité des métiers occupés dans l'entreprise, une pyramide des âges qui s'étale

(3) Le congé de conversion comprend des actions de formation, d'orientation, d'aide aux techniques de recherche d'emploi. Le contrat de travail est suspendu. La durée est de dix mois pour les salarié.e.s âgé.e.s de moins de 50 ans et de seize mois pour les plus de 50 ans. Durant cette période, la personne perçoit $85 \%$ de son salaire brut moyen des douze derniers mois. La convention de conversion a été maintenue de façon dérogatoire dans le cadre du plan social Moulinex et a pris fin en 2001 avec une durée de six mois pour les moins de 50 ans et de dix mois pour les plus de 50 ans. 


\section{Encadré \\ Les temps de l'enquête}

Cette recherche menée dans le cadre d'une thèse en sociologie (RouPNEL, 2007) et financée par l'ANPE (Agence nationale pour l'emploi) et l'Unédic, se découpe en trois grands temps qui correspondent à des séquences temporelles et méthodologiques distinctes ${ }^{(1)}$.

Le premier temps de l'enquête, à l'automne 2002, a été consacré à un travail ethnographique dans les différents sites fermés et au sein des cellules de reclassement. Cette pré-enquête a été l'occasion de rencontrer les différents acteurs intervenant dans l'accompagnement ou la défense des ancien.ne.s Moulinex. La fréquentation hebdomadaire des assemblées générales et réunions a favorisé la réalisation de premiers entretiens sur l'histoire des salarié.e.s, leur présent et leur avenir. Elle a aussi permis la construction d'un questionnaire qui se voulait le plus adapté à l'univers des salarié.e.s et le plus respectueux de la variété des situations vécues. Ce questionnaire comporte huit rubriques thématiques : «Santé», «Logement», «Famille», "Soutien social», "Ressources", "Citoyenneté et identité». II se termine par un calendrier visant à retracer les différentes étapes du parcours de chacun durant les deux années allant de la fin de l'emploi chez Moulinex jusqu'au moment de l'enquête par questionnaire.

La deuxième période d'enquête a démarré en septembre 2003 par la passation du questionnaire. Celle-ci a été réalisée auprès d'un échantillon constitué à partir d'un tirage aléatoire dans le fichier nominatif des 2998 salarié.e.s inscrit.e.s aux cellules de reclassement, transmis par la Mire (Mission interministérielle de revalorisation économique). Cette base de données administrative offrait des renseignements relatifs à l'identité personnelle et sociale de chacun ainsi que des éléments de suivi dans le temps de leur situation professionnelle. Le fichier a d'abord été stratifié à partir de quatre variables principales : sexe (homme/femme), dernier lieu de travail (Alençon, Bayeux, Cormelles, Falaise, Saint-Lô), statut par rapport au marché du travail (actif / inactif - préretraite ou mesures amiante) et niveau de diplôme (niveaux 1 à 4 / niveaux 5 et 6). Ensuite, un individu sur trois a été tiré au sort afin de constituer un échantillon représentatif de la population totale des salarié.e.s licencié.e.s. Après le retrait du fichier des 212 personnes qui, préalablement contactées par courrier, avaient manifesté leur souhait de ne pas participer à l'enquête, 830 ont été rencontrées à leur domicile par trente-neuf enquêteurs (le plus souvent des étudiants et moi-même). Également, des passations de questionnaires ont été enregistrées et retranscrites pour saisir les interactions verbales entre enquêteurs et enquêtés, ce qui a fourni un matériau qualitatif dense.

Enfin, le troisième temps, début 2004, a donné lieu à vingt et un entretiens approfondis auprès d'une souspopulation choisie de manière “raisonnée", c'est-à-dire identifiée comme rencontrant des problèmes d'ordre professionnel, personnel ou social : santé diminuée depuis le licenciement, isolement social, difficultés d'insertion stable sur le marché de l'emploi, etc.

(1) Pour plus de détails sur la méthodologie employée, voir l'annexe 2 de l'ouvrage Les chômeurs de Moulinex (RoupNEL-FuEnTES, 2011).

de 23 à 60 ans et la place des hommes qui représente près de la moitié des 3000 salarié.e.s.

À l'automne 2002, à mon arrivée au sein des rassemblements d'ancien.ne.s salarié.e.s organisés par les syndicalistes de chacune des cinq usines fermées (Alençon, Bayeux, Cormelles, Falaise, Saint-Lô), la disparité des situations que l'on peut constater pour l'ensemble du personnel licencié un an après le plan social m'est apparue clairement dans la composition même des assemblées : une grande partie, pour les plus âgés d'entre eux, a pu bénéficier des dispositifs de mesures d'âge tandis qu'une autre, le plus souvent des femmes et le personnel peu qualifié et non diplômé, se partage entre épisodes de chômage parfois longs et courtes périodes d'emploi (remplacements pour congés maternité ou maladie, assez généralement). Si ces rassemblements sont soupçonnés par les agents de reclassement de freiner la possibilité de se projeter dans un nouvel emploi pour la moitié des ex-salarié.e.s qui y participaient, ils ont en revanche favorisé mes observations et contacts avec beaucoup d'entre eux. Au gré de mes rencontres, ces premiers entretiens m'ont donné accès à leur univers linguistique, à l'articulation entre différents séquençages de leur biographie professionnelle et personnelle, à leurs représentations et projets d'avenir (Demazière, 2003). Mais l'entrée par les assemblées générales et réunions d'ancien.ne.s salarié.e.s ne permet de rencontrer qu'une partie des anciens Moulinex, les personnes ayant retrouvé un emploi et les plus isolées restant inaccessibles. Une enquête par questionnaire deux ans après le licenciement, à l'automne 2003, a permis de parer à ce biais de sélection et d'obtenir une appréhension élargie et longitudinale des conséquences du licenciement (RouPNEL-Fuentes, 2011). L'échantillon a été constitué à partir du fichier de l'ensemble des licencié.e.s inscrit.e.s aux cellules de reclassement et par tirage au sort d'une personne sur trois, soit 830 individus interrogés à leur domicile et en face à face (voir encadré méthodologique). Le traitement statistique des données chiffrées donne un ordre de grandeur des phénomènes étudiés mais aide aussi à découvrir des mécanismes sociaux sous-jacents qui peuvent échapper à la vue de l'observateur 
(Dietrich et al., 2010). Notamment, l'articulation entre les méthodes quantitatives et qualitatives contribue à enrichir des données issues de grandes enquêtes avec des récits de vie de chômeurs pour explorer la construction des projets professionnels entre reconversion et déclassement (Pochic, 2001a). Des entretiens ont été réalisés dans une troisième phase de l'enquête auprès de celles et ceux les plus en difficulté dans leur réinsertion, afin de mieux comprendre les contraintes vécues et les stratégies qu'ils/elles déployaient pour y faire face.

Cette recherche menée auprès des licencié.e.s de Moulinex s'est beaucoup inspirée de celle réalisée auprès des chômeurs de Marienthal, ce village de Basse-Autriche qui, dans les années 1930, avait subi de plein fouet la fermeture de son unique employeur, une manufacture de textile. Le recours ici à différentes méthodologies participe de la même démarche que celle empruntée par Paul Lazarsfeld et son équipe qui visait à relier «l'utilisation d'un matériel chiffré précis à une observation participante» (LAZARSFELD et al., 1981, p. 23). Ces deux enquêtes prennent pour point de départ un événement proche, un licenciement massif et subi, sans réel préavis et dans un même territoire, et ont pour objectif de découvrir l'étendue et la variété de ses conséquences sur la communauté des ex-salarié.e.s, leur santé, leur niveau de vie, leurs relations sociales et leur rapport à la citoyenneté (RouPNEL-FuENTES, 2011).

Le suivi longitudinal du devenir de licenciés a déjà fait l'objet de recherches en sociologie, souvent à partir de panels de chômeurs ou de salariés ayant connu une rupture d'emploi, panels généralement issus de la statistique publique (Pignoni, Poujouly, $1999^{(4)}$; Amossé, Perraudin, Petit, 2012(5)). Vu la complexité technique des enquêtes statistiques longitudinales, le suivi du personnel d'une même entreprise à partir d'enquêtes ad hoc est plus rare telle, notamment, celle menée sur une filature de

(4) Les auteurs s'appuient sur l'enquête conduite par la Direction de l'animation de la recherche, des études et des statistiques (Dares) en 1995-1998, Trajectoires des demandeurs d'emploi et marché local du travail, portant sur une population de 8125 entrants au chômage dans des zones d'emploi du Nord-Pas-de-Calais, d'Île-de-France et de Provence-AlpesCôte-d'Azur. Les résultats de cette enquête sont détaillés dans le numéro spécial "Acteurs locaux de l'emploi» de Travail et emploi en 2000 (Canceill, Pignoni, 2000). L'analyse des parcours de 1752 "chômeurs récurrents», alternant emplois à durée limitée ou en intérim et retour au chômage, révèle une large variété de trajectoires et de profils des personnes concernées.

(5) À partir de l'enquête Formation et qualification professionnelle (FQP) conduite par l'Institut national de la statistique et des études économiques (Insee) en 2003, les auteurs ont sélectionné une sous-population correspondant à 2150000 personnes salariées en 1998 et qui, l'année suivante, ont perdu leur emploi pour démission, fin de contrat ou licenciement. Les auteurs dressent une typologie de trajectoires dessinant différentes mobilités sur le marché du travail : promotion, rotation promotion, stabilité en emploi, emploi-chômage long, rotation déclassement, déclassement et retrait exclusion. coton du Nord de la France (Benarrosh, $\left.1995^{(6)}\right)$. L'enquête auprès des licencié.e.s de Moulinex se distingue de ces travaux en ce qu'elle procède d'une lecture qui, deux ans après la fin de l'emploi, fait le lien avec le passé professionnel dans l'entreprise. Il s'agit alors moins de caractériser les séquences temporelles entre différents cheminements plus ou moins fluides sur le marché du travail que de mettre en regard deux grands pans de l'histoire des ex-Moulinex : un «avant» et un «après» délimités par l'événement du licenciement. Le but est donc de mettre en perspective la perte d'emploi en la resituant dans un continuum temporel incluant l'expérience passée dans l'entreprise et les positions occupées par les ex-salarié.e.s sur le marché $\mathrm{du}$ travail deux ans après. Cette approche révèle que l'analyse des chances d'accéder à une situation professionnelle stable et à temps plein deux ans après le licenciement gagne à prendre en compte les carrières passées chez Moulinex. Pour faire le lien entre l'avant- et l'après-licenciement, la notion de «carrière» sera donc privilégiée à celles de parcours ou de trajectoire. Du fait de la plasticité de ses usages et de l'extension de son champ d'application, elle ne se limite plus désormais aux seuls murs de l'entreprise mais peut aussi s'étendre au marché du travail et même, plus largement, à d'autres types d'activités et de comportements comme le militantisme ou l'anorexie (DARMON, 2008). Grâce à la notion de carrière, il est également possible de saisir à la fois les différentes positions objectivement occupées par les salarié.e.s sur le marché du travail deux ans après leur licenciement et leur perception subjective de cette évolution (Hugues, 1985). Enfin, elle aide à mettre en vis-à-vis le contexte dans lequel a eu lieu le licenciement avec la pluralité des «destins» de licencié.e.s.

C'est donc dans une double perspective, à la fois descriptive et compréhensive, que s'inscrit cet article visant à appréhender les chances différenciées de retour à l'emploi et la diversité des expériences vécues des ex-Moulinex. La première partie, bâtie à partir de modèles de régression, permet de révéler les fortes disparités entre les situations des salarié.e.s deux ans après leur licenciement. Par rapport au constat classique du poids des variables sociodémographiques - sexe, âge, diplôme - sur l'employabilité (Demazière, 2001; MARUANI, 2006), cette étude montre l'intérêt de dépasser la simple distinction entre reclassement et non-reclassement utilisée dans le suivi des ex-Moulinex (RoupNEL,

(6) À partir de grilles de vie, cette enquête, menée en deux vagues (soit vingt-deux mois et seize mois après le licenciement) auprès de 727 licencié.e.s, permet d'identifier plusieurs types d'itinéraires de reclassement par l'examen de l'alternance entre différents événements : accès direct à l'emploi après le licenciement, circulation entre plusieurs emplois temporaires, recours à des dispositifs de conversion ou de formation. 
$\left.2014 \mathrm{a}^{(7)}\right)$, et d'affiner l'analyse avec les caractéristiques de l'emploi retrouvé (stable ou précaire notamment). La deuxième partie sera consacrée à l'exploitation conjointe des différentes variables utilisées précédemment autour d'une analyse factorielle des correspondances multiples (ACM) afin de confronter le passé professionnel des ex-Moulinex avec leur devenir deux ans après le licenciement et, ce faisant, d'identifier différents types de carrières qui seront présentés plus en détail à l'aide d'entretiens approfondis dans la dernière partie. La particularité de cette enquête est également d'accorder une attention à la localisation géographique puisque l'entreprise possédait cinq sites de production au sein de la région Basse-Normandie. Cette donnée fournit des éléments relatifs à la fois au marché de l'emploi et à l'aspect «concentratif» de la mise au chômage dans certains espaces : le département du Calvados a en effet subi la fermeture de trois usines qui représentaient les deux tiers des salarié.e.s licencié.e.s, qui se sont retrouvé.e.s en situation de concurrence les un.e.s par rapport aux autres sur un bassin d'emploi sinistré.

\section{Le devenir des licencié.e.s de Moulinex : des trajectoires contrastées}

Que sont devenu.e.s les licencié.e.s de Moulinex en septembre 2003, c'est-à-dire à la fin des mesures de conversion? Au fondement de cette recherche, se trouve la volonté de dépasser l'idée uniformisante du licenciement comme d'un événement décliné presque uniquement sur le registre de la perte, du choc (Trotzier, 2006) ou du déclassement. Dans Perte d'emploi, perte de soi, Danièle Linhart et ses coauteurs (2002) montrent que la rupture subie par les salarié.e.s de l'usine Chausson de Creil n'est pas que professionnelle, elle se vit aussi personnellement. $\mathrm{Si}$, de la même manière, le licenciement des Moulinex a été synonyme pour beaucoup d'anéantissement ou de déchéance, il a pu sonner pour certains et certaines comme une délivrance, l'arrêt d'un travail épuisant et se présenter comme une opportunité pour «passer à autre chose», une invitation à un nouveau départ professionnel. Vécu comme une véritable césure dans une carrière professionnelle ininterrompue jusqu'alors, le licenciement peut aussi constituer une transition, une porte d'entrée vers un nouvel emploi.

(7) L'objet du chapitre intitulé «Les cadres sociaux du reclassement» est de porter un regard critique sur ce terme en en révélant les différents usages et la variété des réalités qu'il peut recouvrir. Être considéré comme reclassé dans le cadre du suivi des ancien.ne.s salarié.e.s de Moulinex ne s'appliquait pas seulement aux personnes ayant retrouvé un emploi. Il renvoyait aussi à celles concernées par une "solution», qu'elle soit d'âge (préretraites), de formation ou sociale (congés maladie ou projets de vie) qui n'ont pas pour but une réinsertion professionnelle immédiate.
La particularité de la recherche menée auprès de 830 ex-Moulinex est d'inscrire l'événement du licenciement dans une perspective longitudinale en interrogeant les liens entre passé dans l'entreprise et situations retrouvées deux ans après le licenciement. Tout en esquissant certains profils sociaux de salarié.e.s, les trois trajectoires typiques observées deux ans après le licenciement vont être présentées successivement.

La première concerne $38 \%$ des licencié.e.s enquêté.e.s, qui ont pu bénéficier d'une des mesures d'âge proposées par le plan social (préretraites, dispositifs d'âge) au cours des deux années écoulées depuis le licenciement ou qui étaient, au moment de l'enquête, en attente d'une de celles-ci ${ }^{(8)}$. Sans surprise, le personnel le plus âgé et le plus ancien dans l'emploi y est fortement représenté puisque l'accès à la préretraite AS-FNE n'était ouvert qu'aux personnes ayant plus de 57 ans et, de façon dérogatoire, à celles âgées de 56 ans; la préretraite amiante ne pouvait s'appliquer qu'aux salarié.e.s de plus de 50 ans mais de moins de 57 ou 56 ans. En outre, seules les personnes ayant totalisé 160 trimestres de cotisation dans les régimes de base obligatoires de l'assurance vieillesse pouvaient prétendre à l'allocation équivalent retraite (AER) ou à l'allocation chômeurs âgés (ACA). Enfin, c'est aussi sous condition d'âge qu'une dispense de recherche d'emploi a pu être accordée à certains chômeurs. Les mesures d'âge ont, d'une certaine manière, permis de retirer du marché du travail les salarié.e.s dont l'âge et l'ancienneté professionnelle étaient les plus élevés. Par ailleurs, avoir suivi une ou plusieurs formations internes diminuait les possibilités de faire l'objet de mesures d'âge. En effet, pour le droit à la retraite, les périodes de formation professionnelle n'étaient pas validées au même titre que des périodes d'activités ${ }^{(9)}$. De même, l'accès à la préretraite amiante dépendait fortement de la durée d'exposition au produit, durée qui n'était pas la même selon les sites de production. Les salarié.e.s des usines d'Alençon, de Bayeux et de Cormelles-le-Royal eurent trois fois moins de chances de prétendre à une mesure d'âge que ceux et celles de Saint-Lô, « toutes choses égales par ailleurs» (voir tableau 1). Le cas de la préretraite amiante est emblématique d'une mesure de santé publique dont le but premier (protéger les individus d'une substance toxique) a été détourné pour être utilisé comme mesure sociale de lutte contre le chômage. D'où cette phrase ambiguë souvent prononcée par des personnes préretraitées à propos de leur situation actuelle: "Moi, je n'ai

(8) Ont été considérées comme «en attente de mesures d'âge», les personnes pouvant prétendre à l'une d'entre elles dans les deux années qui ont suivi l'enquête (c'est-à-dire les années 2004 ou 2005).

(9) Les cotisations à la retraite des stagiaires de la formation professionnelle sont calculées sur une base forfaitaire qui est de six fois inférieure au salaire minimum interprofessionnel de croissance (Smic). 
Tableau 1 : Le profil des ex-salarié.e.s concerné.e.s par une mesure d’âge

\begin{tabular}{|c|c|c|c|}
\hline Variable & $\begin{array}{c}\text { Odds ratio } \\
\text { (vs non concerné.e.s) }\end{array}$ & Effectifs & Pourcentage \\
\hline \multicolumn{4}{|l|}{ Modalités } \\
\hline \multicolumn{4}{|l|}{ Sexe } \\
\hline Homme & 1,09 & 368 & 44,34 \\
\hline Femme & Réf. & 462 & 55,66 \\
\hline \multicolumn{4}{|l|}{ Âge au moment de l'enquête } \\
\hline 39 ans et moins & Réf. & 84 & 10,12 \\
\hline 40 ans à 49 ans & 0,88 & 312 & 37,59 \\
\hline 50 ans et plus & $20,28 * * *$ & 434 & 52,29 \\
\hline \multicolumn{4}{|l|}{ Ancienneté chez Moulinex } \\
\hline 19 années et moins & Réf. & 183 & 22,05 \\
\hline 20 années à 29 années & $3,25 * * *$ & 299 & 36,02 \\
\hline 30 années et plus & $5,01 * * *$ & 348 & 41,93 \\
\hline \multicolumn{4}{|l|}{ Dernier diplôme obtenu } \\
\hline Aucun [1] & Réf. & 480 & 57,83 \\
\hline $\begin{array}{l}\text { CAP-BEP (certificat d'aptitude professionnelle - brevet } \\
\text { d'études professionnelles) }\end{array}$ & 0,77 & 245 & 29,52 \\
\hline Bac et plus & 1,89 & 77 & 9,28 \\
\hline \multicolumn{4}{|l|}{ Dernière position professionnelle chez Moulinex } \\
\hline Agent de production & 0,65 & 346 & 41,69 \\
\hline Agent de production - ouvrier qualifié & 0,81 & 175 & 21,08 \\
\hline Ouvrier qualifié & 0,5 & 67 & 8,07 \\
\hline Employé, technicien, agent de maîtrise & 0,76 & 211 & 25,42 \\
\hline Cadre et ingénieur & Réf. & 31 & 3,73 \\
\hline \multicolumn{4}{|l|}{ Formation suivie chez Moulinex } \\
\hline De 0 à 2 formations & Réf. & 443 & 53,37 \\
\hline De 3 à 6 formations & $0,58 * *$ & 387 & 46,63 \\
\hline \multicolumn{4}{|l|}{ Site } \\
\hline Alençon & $0,35 * *$ & 221 & 26,63 \\
\hline Bayeux & $0,31 * *$ & 89 & 10,72 \\
\hline Cormelles (Caen) & $0,35 * *$ & 379 & 45,66 \\
\hline Falaise & 0,51 & 92 & 11,08 \\
\hline Saint-Lô & Réf. & 49 & 5,9 \\
\hline \multicolumn{4}{|l|}{ Vie conjugale et situation du conjoint } \\
\hline En couple et conjoint en emploi ou en mesure d'âge & 0,96 & 598 & 72,05 \\
\hline En couple et conjoint sans emploi & 1,09 & 91 & 10,96 \\
\hline Seul & Réf. & 134 & 16,14 \\
\hline \multicolumn{4}{|l|}{ Adhésion - mandat syndical } \\
\hline Aucune & Réf. & 588 & 70,84 \\
\hline Au moins une [2] & 0,93 & 242 & 29,16 \\
\hline Effectifs & & 830 & 100 \\
\hline Détails effectifs & & $275 / 555$ & $33,13 / 66,86$ \\
\hline
\end{tabular}

Odds ratios et degrés de significativité $*$ : p. $<, 1 ; * *:$ p. $<, 05 ; * * *:$ p $<, 001$

[1] Sans diplôme : sans diplôme, certificat d'étude primaire (CEP), brevet des collèges (BEPC : brevet d'études du premier cycle du second degré).

[2] Adhésion à au moins un de ces statuts : salarié protégé, délégué du personnel, délégué syndical, adhérent.

Lecture : Toutes choses égales par ailleurs, la probabilité d'avoir été concerné par une mesure d'âge (plutôt que de ne pas l'avoir été) est 20,28 fois supérieure pour les 50 ans ou plus que pour les 39 ans ou moins.

Champ : Ensemble des personnes enquêtées.

Source : Enquête Moulinex, 2003, 830 individus.

pas à rechercher d'emploi car heureusement j'ai l'amiante!» Enfin, bien que de façon non significative d'un point de vue statistique, il faut noter le rapport de chances plus élevé d'entrées en mesure d'âge pour les hommes, les détenteurs d'un diplôme supérieur ou égal au baccalauréat et les cadres ou ingénieurs. Ce point est à relier au fait que ces catégories sont davantage protégées des épisodes de chômage passés durant leur carrière professionnelle.
Le deuxième type de trajectoire est celui ayant conduit au retour à l'emploi, en septembre 2003. Il concerne $32 \%$ des personnes enquêtées, dont seule la moitié bénéficie d'une insertion professionnelle stable (soit un contrat à durée indéterminée [CDI] ou un emploi indépendant) et à temps complet. À titre de comparaison, une étude menée à partir des statistiques nationales de la Dares (BobBio et al., 2009) sur les sorties de mesures de reclassement (contrat de transition professionnelle ou 
Tableau 2 : Le profil des ex-salarié.e.s ayant retrouvé un emploi deux ans après

\begin{tabular}{|c|c|c|c|}
\hline Variable & $\begin{array}{c}\text { Odds ratio } \\
\text { (vs au chômage) }\end{array}$ & Effectifs & Pourcentage \\
\hline \multicolumn{4}{|l|}{ Modalités } \\
\hline \multicolumn{4}{|l|}{ Sexe } \\
\hline Homme & $1,73 * *$ & 301 & 54,23 \\
\hline Femme & Réf. & 254 & 45,77 \\
\hline \multicolumn{4}{|l|}{ Âge au moment de l'enquête } \\
\hline 39 ans et moins & $2,5 * *$ & 82 & 14,77 \\
\hline 40 ans à 49 ans & $1,78 * * *$ & 295 & 53,15 \\
\hline 50 ans et plus & Réf. & 178 & 32,07 \\
\hline \multicolumn{4}{|l|}{ Ancienneté chez Moulinex } \\
\hline 19 années et moins & Réf. & 170 & 30,63 \\
\hline 20 années à 29 années & 0,7 & 218 & 39,28 \\
\hline 30 années et plus & 1,01 & 167 & 30,09 \\
\hline \multicolumn{4}{|l|}{ Dernier diplôme obtenu } \\
\hline Aucun [1] & 0,76 & 301 & 54,23 \\
\hline CAP-BEP & 0,62 & 176 & 31,71 \\
\hline Bac et plus & Réf. & 56 & 10,09 \\
\hline \multicolumn{4}{|l|}{ Dernière position professionnelle chez Moulinex } \\
\hline Agent de production & 1,08 & 224 & 40,36 \\
\hline Agent de production - ouvrier qualifié & 1,35 & 120 & 21,62 \\
\hline Ouvrier qualifié & 1,43 & 51 & 9,19 \\
\hline Employé, technicien, agent de maîtrise & 1,31 & 139 & 25,05 \\
\hline Cadre et ingénieur & Réf. & 21 & 3,78 \\
\hline \multicolumn{4}{|l|}{ Formation suivie chez Moulinex } \\
\hline 2 et moins & Réf. & 285 & 51,35 \\
\hline De 3 à 6 & 1,06 & 270 & 48,85 \\
\hline \multicolumn{4}{|l|}{ Site } \\
\hline Alençon & Réf. & 143 & 25,77 \\
\hline Bayeux & $0,26 * * *$ & 70 & 12,61 \\
\hline Cormelles (Caen) & $0,36 * * *$ & 258 & 46,49 \\
\hline Falaise & $0,42 * *$ & 55 & 9,91 \\
\hline Saint-Lô & 0,77 & 29 & 5,23 \\
\hline \multicolumn{4}{|l|}{ Vie conjugale et situation du conjoint } \\
\hline En couple et conjoint en emploi ou en mesure d'âge & 1,12 & 412 & 74,23 \\
\hline En couple et conjoint sans emploi & 1,27 & 54 & 9,73 \\
\hline Seul & Réf. & 86 & 15,5 \\
\hline \multicolumn{4}{|l|}{ Adhésion - mandat syndical } \\
\hline Aucune & Réf. & 402 & 72,43 \\
\hline Au moins une [2] & 1,28 & 153 & 27,57 \\
\hline Effectifs & & 555 & \\
\hline Détails effectifs & & $296 / 259$ & $53,33 / 46,67$ \\
\hline
\end{tabular}

Odds ratios et degrés de significativité $*:$ p. $<, 1 ; * *:$ p. $<, 05 ; * * *: p<, 001$

[1] Sans diplôme : sans diplôme, certificat d'étude primaire (CEP), brevet des collèges (BEPC).

[2] Adhésion à au moins un de ces statuts : salarié protégé, délégué du personnel, délégué syndical, adhérent.

Lecture : Toutes choses égales par ailleurs, la probabilité d'avoir retrouvé un emploi deux an après est 1,73 fois supérieure pour les hommes que pour les femmes.

Champ : Ensemble des personnes actives à la date d'enquête.

Source : Enquête Moulinex, 2003, 555 individus.

convention de reclassement personnalisé) deux ans après un licenciement fournit des résultats bien différents puisque deux tiers des personnes licenciées au second semestre 2006 sont en emploi début 2008, soit près du double des ex-Moulinex sur la même durée. Quelles sont les raisons du handicap subi par les licencié.e.s du fabricant d'électroménager dans leur retour à l'emploi ? On retrouve ici les inégalités sociales en termes d'employabilité (ou de recrutabilité, voire de discriminations à l'embauche), avec le fort désavantage accusé par les femmes, le personnel âgé et peu qualifié. Le rôle du marché local de l'emploi est également notable, les salarié.e.s du département le plus touché par les pertes d'emplois (ici le Calvados) ayant plus de difficultés que les autres à retrouver un emploi «toutes choses égales par ailleurs» (voir tableau 2).

Notons d'abord que près des trois quarts des ex-Moulinex concernés par un retour sur le marché du travail ont plus de 45 ans au moment de l'enquête. Or, si un âge élevé est une condition nécessaire pour 
Tableau 3 : Le profil des ex-salarié.e.s ayant retrouvé un emploi stable à temps plein

\begin{tabular}{|c|c|c|c|}
\hline Variable & $\begin{array}{l}\text { Odds ratio (vs en } \\
\text { emploi instable et/ } \\
\text { ou à temps partiel }\end{array}$ & Effectifs & Pourcentage \\
\hline \multicolumn{4}{|l|}{ Modalités } \\
\hline \multicolumn{4}{|l|}{ Sexe } \\
\hline Homme & $2,34 * * *$ & 158 & 53,38 \\
\hline Femme & Réf. & 138 & 46,62 \\
\hline \multicolumn{4}{|l|}{ Âge au moment de l'enquête } \\
\hline 39 ans et moins & $2,45^{*}$ & 57 & 19,26 \\
\hline 40 ans à 49 ans & $2,56^{* *}$ & 160 & 54,05 \\
\hline 50 ans et plus & Réf. & 79 & 26,69 \\
\hline \multicolumn{4}{|l|}{ Ancienneté chez Moulinex } \\
\hline 19 années et moins & Réf. & 108 & 36,49 \\
\hline 20 années à 29 années & 0,68 & 105 & 35,47 \\
\hline 30 années et plus & 0,69 & 83 & 28,04 \\
\hline \multicolumn{4}{|l|}{ Dernier diplôme obtenu } \\
\hline Aucun [1] & 0,59 & 146 & 49,32 \\
\hline CAP-BEP & 0,85 & 96 & 32,43 \\
\hline Bac et plus & Réf. & 38 & 12,84 \\
\hline \multicolumn{4}{|l|}{ Dernière position professionnelle chez Moulinex } \\
\hline Agent de production & 0,46 & 100 & 33,78 \\
\hline Agent de production - ouvrier qualifié & $1,39 * * *$ & 66 & 22,3 \\
\hline Ouvrier qualifié & $2,39 * * *$ & 33 & 11,15 \\
\hline Employé, technicien, agent de maîtrise & $1,2 * *$ & 84 & 28,38 \\
\hline Cadre et ingénieur & Réf. & 13 & 4,39 \\
\hline \multicolumn{4}{|l|}{ Formation suivie chez Moulinex } \\
\hline 2 et moins & Réf. & 141 & 47,64 \\
\hline De 3 à 6 & 1,05 & 155 & 52,36 \\
\hline \multicolumn{4}{|l|}{ Site } \\
\hline Alençon & Réf. & 100 & 33,78 \\
\hline Bayeux - Falaise - Saint-Lô & 0,6 & 72 & 24,33 \\
\hline Cormelles (Caen) & $0,47 * *$ & 124 & 41,89 \\
\hline \multicolumn{4}{|l|}{ Vie conjugale et situation du conjoint } \\
\hline En couple et conjoint en emploi ou en mesure d'âge & $2,29 *$ & 226 & 76,35 \\
\hline En couple et conjoint au chômage & 2,25 & 29 & 9,8 \\
\hline Seul & Réf. & 39 & 13,18 \\
\hline \multicolumn{4}{|l|}{ Adhésion - mandat syndical } \\
\hline Aucune & Réf. & 220 & 74,32 \\
\hline Au moins une [2] & 0,92 & 76 & 25,68 \\
\hline Total effectifs & & 296 & \\
\hline Détails effectifs & & $137 / 159$ & $46,28 / 53,72$ \\
\hline
\end{tabular}

Odds ratios et degrés de significativité $*$ : p. $<, 1 ; * *:$ p. $<, 05 ; * * *: p<, 001$

[1] Sans diplôme : sans diplôme, certificat d'étude primaire (CEP), brevet des collèges (BEPC).

[2] Adhésion à au moins un de ces statuts : salarié protégé, délégué du personnel, délégué syndical, adhérent.

Lecture : Toutes choses égales par ailleurs, la probabilité d'avoir retrouvé un emploi stable à temps plein est 2,34 fois supérieure pour les hommes que pour les femmes.

Champ : Ensemble des personnes en emploi à la date d'enquête.

Source : Enquête Moulinex, 2003, 296 individus.

prétendre à une mesure d'âge, il constitue une caractéristique défavorable pour le retour à l'emploi (voir tableau 2) et, de surcroît, pour l'emploi durable et à temps complet (voir tableau 3). Par rapport aux personnes de plus de 50 ans, celles de moins de 40 ans sont, "toutes choses égales par ailleurs», 2,5 fois plus souvent en emploi qu'au chômage et celui-ci a alors 2,6 fois plus de chances d'être stable et à temps plein. Les difficultés des seniors sur le marché de l'emploi se retrouvent ici (JoLIVET, 2003; Amossé et al., 2012), accompagnées des stéréotypes accolés au personnel vieillissant supposé moins flexible ou en mauvaise santé, qui ont intrinsèquement peu à voir avec l'âge à proprement parler. Beaucoup d'enquêtés rapportent la réticence clairement exprimée par des employeurs à embaucher un personnel âgé et déplorent que leurs compétences et expériences professionnelles ne soient pas mieux prises en compte.

Ensuite, si hommes et femmes sont à égalité devant la sortie du marché du travail via des mesures d'âge, le retour à l'emploi est par contre fortement discriminant selon le sexe. Alors qu'elles représentaient $54 \%$ des ex-Moulinex au moment 
du licenciement, les femmes n'étaient plus qu'un tiers à être parvenues à se réinsérer deux ans après. «Toutes choses égales par ailleurs», elles ont deux fois moins de chances d'avoir retrouvé un emploi que les hommes (voir tableau 2) et, quand elles y parviennent, celui-ci a 2,3 fois plus de risques d'être à durée limitée et à temps partiel (voir tableau 3). Existe-t-il une discrimination à l'encontre des femmes ou bien le marché de l'emploi local est-il moins bien pourvu en emplois considérés comme féminins ? Dans les entretiens approfondis, peu de candidates à l'embauche interrogées ont déclaré se sentir pénalisées sur le marché du travail en raison de leur sexe. Toutefois, la recherche d'emploi a conduit un certain nombre d'entre elles à envisager une reconversion en dehors du monde de l'industrie, vers des emplois considérés comme masculins (ambulancier, chauffeur de bus, etc.) ou dans le secteur des services à la personne. Les reconversions professionnelles vers ce type de postes sont, elles, beaucoup moins répandues parmi les hommes.

Enfin, le marché local du travail influence fortement l'employabilité puisque les chances de retrouver un emploi ne sont pas distribuées de manière égale selon la localisation géographique. On compte ainsi $41 \%$ des salarié.e.s d'Alençon dans l'Orne parvenu.e.s à se réinsérer, contre seulement $24 \%$ de ceux de Cormelles-le-Royal. «Toutes choses égales par ailleurs», les employé.e.s des trois usines de Bayeux, Cormelles-le-Royal et Falaise encourent jusqu'à quatre fois plus de risques que ceux d'Alençon d'être sans emploi deux ans après la fermeture (voir tableau 2). Comment expliquer ce différentiel si élevé ? Ces trois villes du Calvados se situent toutes à l'intérieur d'un périmètre restreint et encadré par, au nord, une façade maritime et, au sud, ainsi qu'à l'ouest, deux départements également touchés par la fermeture de Moulinex (l'Orne et la Manche). Les salariés d'Alençon ont, quant à eux, pu étendre leur champ de prospection vers les départements limitrophes de la Sarthe et de la Mayenne de la région des Pays de la Loire. Il est à noter aussi que le paysage industriel et la dynamique du marché du travail autour de Cormelles-le-Royal et Alençon sont bien différents ${ }^{(10)}$. Alors qu'en périphérie de Caen se trouvent implantées de longue date de grandes industries embauchant presque uniquement du personnel qualifié de techniciens et d'ingénieurs dans l'industrie (PSA, Bosch, etc.), le sud de l'Orne, lui, se caractérise par un tissu de jeunes PME (petites et moyennes entreprises) recrutant un plus large spectre de travailleurs, pour des contrats de travail plus souvent pérennes. D'ailleurs, «toutes choses égales par ailleurs», la réinsertion professionnelle est deux fois plus souvent assurée et à temps plein à Alençon qu'à Cormelles-le-Royal (voir tableau 3).

(10) Alors que le taux de chômage dans le Calvados s'établissait en 2001 à $13,5 \%$, il n'était que de $11,5 \%$ dans la Manche et $11,7 \%$ dans l'Orne.
Si les femmes, les salarié.e.s les plus âgé.e.s et ceux appartenant aux usines du Calvados présentent de plus faibles chances de se retrouver en emploi deux ans après le licenciement, le personnel syndiqué, ancien et formé chez Moulinex, mais aussi le moins diplômé et qualifié, ne se retrouve pas plus désavantagé que les autres. Ces derniers résultats divergent de ceux de la littérature sur les chômeurs et les licenciés pour motif économique qui mettent en avant notamment l'effet négatif du temps passé dans une organisation ainsi que le rôle favorable du diplôme initial et du niveau d'éducation pour le retour à l'emploi (Ledrut, 1966; Demazière, 1995 ; MARGolis, 2002; Amossé et al., 2012).

Avec le temps, les diplômes perdent-ils de leur valeur sur le marché du travail ? Ou bien les qualifications souffrent-elles d'une obsolescence ? Cette première question invite à étudier les deux années écoulées depuis le licenciement. En retraçant l'évolution du taux de chômage par catégorie de sexe et de diplômes, l'effet de ce dernier apparaît immédiatement à l'entrée dans les dispositifs de conversion, c'est-à-dire en janvier 2002 (voir graphique). En effet, les courbes des hommes et des femmes peu diplômés et celles des diplômés se distinguent très nettement jusqu'en mai 2002 . À cette date, la courbe des femmes diplômées se rapproche de celles des non diplômés puis c'est la courbe des hommes non diplômés qui entame alors une inflexion jusqu'à rejoindre celles des diplômés (hommes et femmes). Ce mouvement indique clairement que l'absence de diplôme handicape «à terme» bien plus les femmes que les hommes. Si les variables du sexe, de l'âge et de localisation jouent sur les chances de retour à l'emploi sur une longue période, l'effet «signal» du diplôme est, lui, de courte durée. Il s'efface au fil du temps et vient renforcer les inégalités sexuelles.

\section{Graphique : Évolution du taux de chômage} par catégorie de sexe et de diplôme

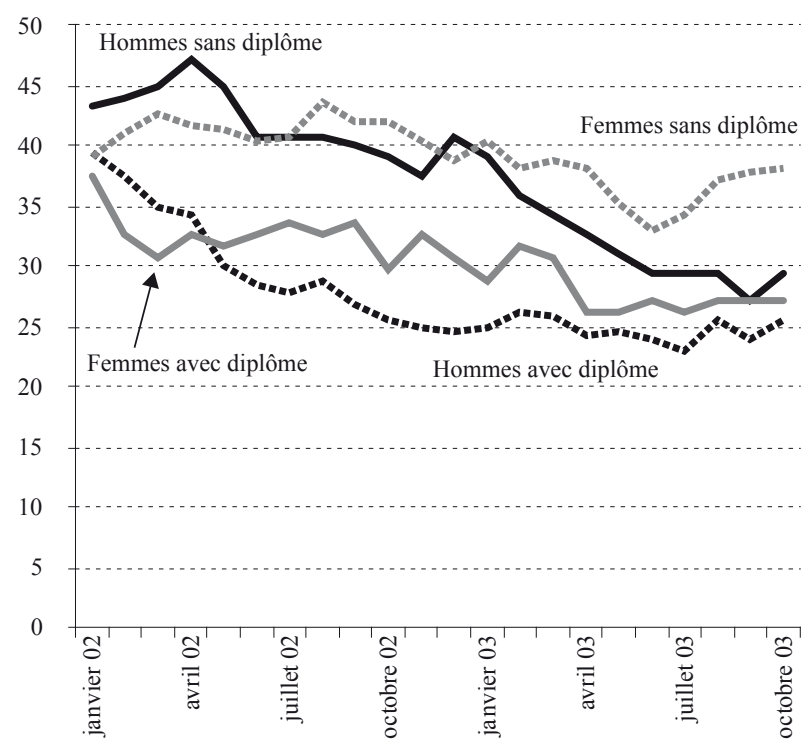

Source : Enquête Moulinex, 2003, 830 individus. 
Ceci nous amène à parler plus spécifiquement d'un troisième type de trajectoire qui concerne $16 \%$ des licencié.e.s ${ }^{(11)}$, celui conduisant à une insertion stable et à temps complet. Si la catégorie professionnelle n'intervient pas dans les chances de rester sur le marché du travail, elle influe en revanche sur la forme de l'emploi retrouvé (voir tableau 3). Deux ans après le licenciement, alors que $80 \%$ des ouvriers qualifiés ont retrouvé un emploi stable et à temps complet, cette situation n'a concerné que $21 \%$ des agents de production (nouvelle appellation managériale pour les ouvriers spécialisés [OS]). Il semble donc que la dernière position professionnelle occupée chez Moulinex protège de l'emploi à durée déterminée et donc, à terme, du chômage ainsi que d'un emploi à temps partiel et donc moins rémunéré. Monsieur Allard ${ }^{(12)}$ était ouvrier qualifié dans l'usine de Cormelles-le-Royal. Ayant 53 ans au moment de l'entretien, il n'avait pas encore retrouvé un emploi mais savait que sa qualification lui permettrait de résister au déclassement représenté par les «petits boulots» que la cellule emploi lui a proposés pour se réinsérer rapidement.

"C'est vrai que s'ils me proposent un boulot où je suis payé 5000 balles, c'est pas motivant. [...] Les tarifs, c'est 6,83 euros, ça fait 44 balles à l'heure, c'est pas des boulots intéressants, des boulots à la ramasse, avec un salaire à la ramasse, $j$ 'ai pas envie de travailler à coups de trique.»

(Monsieur Allard, 53 ans, en couple, ouvrier qualifié chez Moulinex, Cormelles, sans emploi à la recherche d'un emploi.)

Le personnel ayant suivi des formations internes à Moulinex ne se retrouve pas plus désavantagé que les autres alors qu'on pourrait s'attendre à ce que ce capital professionnel, constitué dans et pour l'entreprise, vienne jouer défavorablement dans leur retour à l'emploi. Certaines formations internes ont favorisé l'acquisition de compétences, certes spécifiques, mais recherchées dans d'autres industries et, notamment, l'industrie d'équipement (automobile par exemple). Elles ont surtout permis à leur détenteur de se distinguer des autres candidats à l'embauche. Alors que l'ancienneté professionnelle dans l'entreprise et l'adhésion syndicale sont perçues comme des freins à l'emploi (MARGOLIS, 2002), ces attributs ont pu au contraire être pourvoyeurs de «liens faibles» (GRANOVETTER, 1974) et parfois favoriser la constitution de groupes d'entraide informels en vue de reclassements dans des entreprises avoisinantes ${ }^{(13)}$. Le cas des cadres est à part. Leur très faible nombre dans l'échantillon (huit cadres administratifs et commerciaux et vingt-trois ingénieurs) s'explique par le départ d'une grande

(11) Soit la moitié des personnes en emploi en 2003.

(12) Les noms de famille des personnes dont des extraits de discours ont été retenus ici sont fictifs.

(13) Notons qu'il n'existait pas chez Moulinex de syndicat majoritaire mais, au contraire, un front syndical très éclaté. partie d'entre eux avant même le licenciement. Déjà minoritaires dans l'entreprise, ils avaient eu vent des inquiétants problèmes que traversait Moulinex et avaient rapidement entamé par anticipation des recherches d'emploi, et n'étaient donc plus qu'une poignée, ceux qui n'avaient pu partir, au moment de la liquidation judiciaire.

En suivant le devenir des licencié.e.s deux ans après leur licenciement, trois situations ont donc pu être clairement identifiées : la première est celle des plus âgé.e.s et plus ancien.ne.s dans l'emploi qui ont pu prétendre immédiatement ou dans les années suivantes à l'une des mesures d'âge proposées par le plan social; la deuxième est emblématique du parcours du combattant du personnel féminin ${ }^{(14)}$, d'âge «intermédiaire» ${ }^{(15)}$ et issu des usines Moulinex du Calvados et que leur faible qualification soumet plus fortement au chômage, à l'emploi précaire et à temps partiel; la dernière trajectoire, enfin, correspond à celle des hommes, jeunes et qualifiés, et des salariés d'Alençon parvenus à se réinsérer de façon pérenne.

Les difficultés rencontrées par les femmes, le personnel âgé et venant des usines du Calvados révèlent que des effets de sélection des candidat.e.s à l'embauche sont à l'œuvre sur le marché du travail local qui souffre d'une pénurie d'emplois et qui est donc plus enclin à embaucher un personnel jeune, masculin et qualifié. Mais les inégalités de sexe, d'âge et de localisation géographique ne trouvent-elles pas aussi leur origine dans le passé professionnel chez Moulinex?

\section{L'espace professionnel de l'entreprise toujours présent après sa fermeture}

L'exploration, deux ans après le licenciement, du devenir des ex-Moulinex permet de distinguer trois types de positions vis-à-vis du marché du travail : les sorties définitives de celui-ci, les situations professionnelles précaires ou de chômage et, au contraire, des retours durables dans l'emploi. Peut-on alors entrevoir une correspondance entre la segmentation repérée dans les trajectoires ultérieures au licenciement et la répartition du personnel dans l'espace professionnel de Moulinex ? Pour cela, un retour sur l'histoire de l'entreprise et sa politique de recrutement pourra éclairer les différentes expériences professionnelles vécues et fournira ainsi un faisceau explicatif supplémentaire des difficultés rencontrées par les ex-salarié.e.s. Le

(14) Pour en savoir plus, voir RouPnel-Fuentes (2014b).

(15) Ce terme a été choisi pour désigner les personnes considérées comme trop âgées pour retrouver un emploi facilement mais trop jeunes pour prétendre à l'une des mesures d'âge prévues par le plan social. 


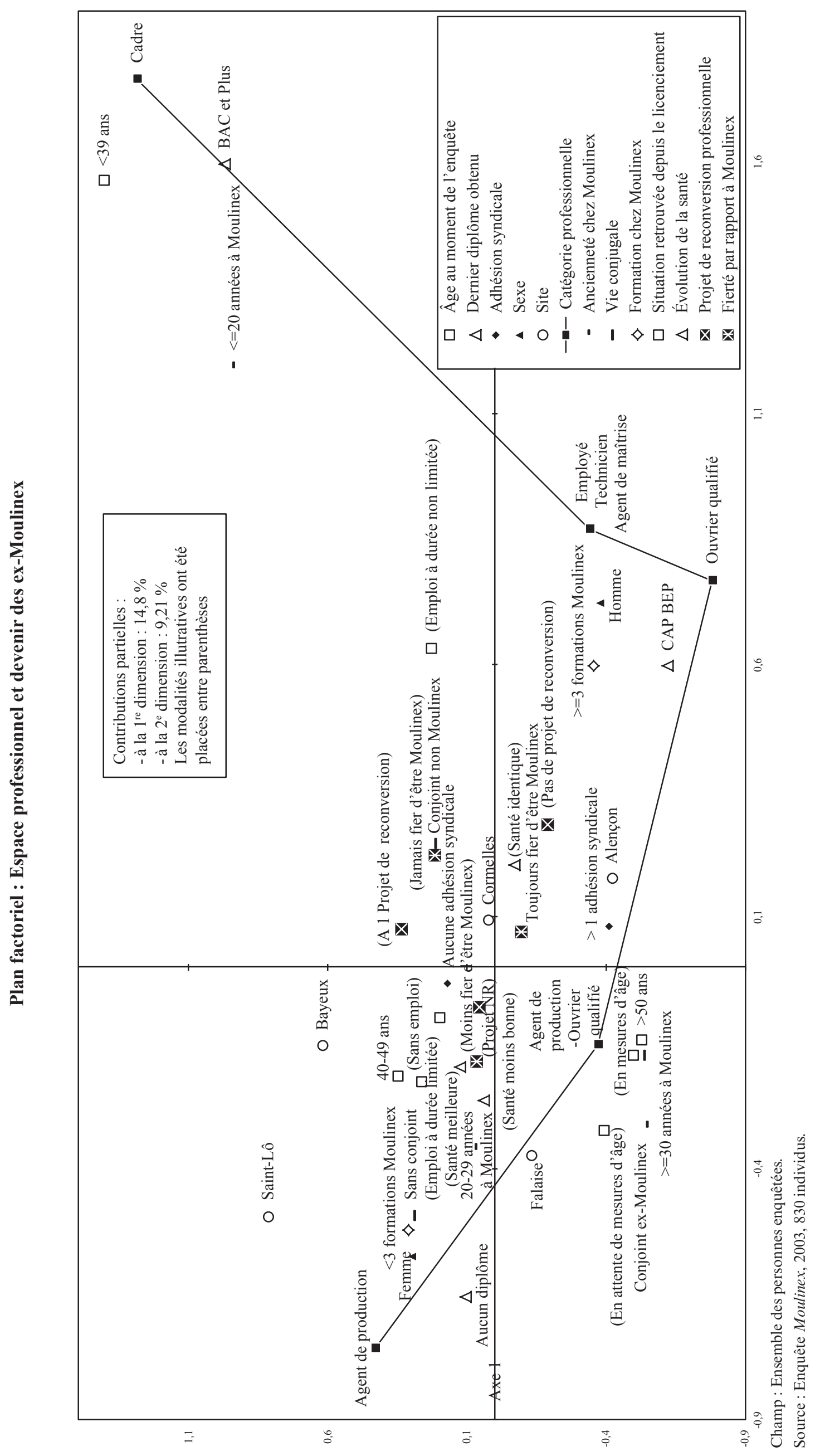

- 28 - Travail et Emploi $n^{\circ} 138$ 
contexte social et professionnel de Moulinex, au moment de sa prospérité et dans le temps des restructurations (1998-2003), est développé dans RouPNEL-FuENTES (2011) dont la première partie est consacrée à l'histoire du fabricant bas-normand de petit électroménager.

Alors que les traitements statistiques utilisés jusqu'alors ont servi à identifier des profils sociaux de licencié.e.s à partir de leurs caractéristiques «toutes choses égales par ailleurs », ceux-ci vont désormais permettre d'explorer les parcours chez Moulinex en prenant conjointement en compte les variables à l'aide d'une analyse factorielle des correspondances multiples (ACM; voir plan factoriel et tableau des contributions aux axes des modalités actives en annexe). La proximité de certaines modalités mettra alors en lumière des zones de cumul des caractéristiques freinant le retour à l'emploi tandis que leurs oppositions indiqueront les lignes de partage qui traversent la population des ex-salarié.e.s de Moulinex.

Pour l'analyse des correspondances multiples, le choix retenu a été de placer les variables explicatives utilisées dans les modèles de régression précédents comme variables actives, c'est-à-dire comme intervenant sur la structuration et la définition des dimensions, le but n'étant pas ici de rechercher les motifs déterminant le retour à l'emploi mais de dresser l'espace professionnel chez Moulinex et, ce faisant, les conditions dans lesquelles se trouvaient les salarié.e.s au moment de la perte de leur emploi. La projection des variables supplémentaires reflétant le devenir des licencié.e.s vise à faire correspondre cet espace social et professionnel à la variété des situations retrouvées et vécues deux ans après le licenciement. Aussi, la distinction entre emploi précaire et emploi stable ou assuré, sera conservée dans cette analyse.

Le plan factoriel fait alors apparaître une nette partition entre deux pôles: l'un regroupant à gauche les modalités «Femme», «Agent de production», «40-49 ans», «20-29 années à Moulinex», "Aucun diplôme» et un autre, situé à droite, rassemblant les modalités «Homme», «CAP BEP», «Ouvrier qualifié», «Employé, technicien, agent

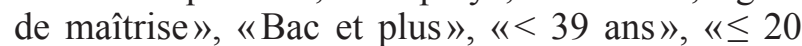
années à Moulinex». Cette opposition portée par le premier axe, c'est-à-dire par l'axe horizontal, cristallise la division du travail longtemps effective chez Moulinex entre, d'un côté, la sphère de l'exécution du travail, caractérisée par un personnel féminin, peu qualifié, d'un âge et d'une ancienneté professionnelle «intermédiaires» et, de l'autre, le pôle de la conception du travail représenté par des travailleurs masculins, qualifiés (techniciens et maîtrise), plus jeunes et moins anciens chez Moulinex.

Cette division est le fruit d'une politique d'embauche systématique, jusque dans les années 1980, d'une main-d'œuvre peu diplômée et surtout féminine, vouée à occuper des postes de production. Ceci explique aussi pourquoi, au moment du licenciement, les trois quarts des femmes n'ont pas de diplôme et se situent dans la tranche d'âge allant de 46 à 52 ans. Mais pour répondre au besoin d'innovation et de technicité de ses produits, Moulinex a fait également appel à un personnel qualifié et diplômé, massivement masculin, attiré par la politique salariale de l'entreprise et les perspectives de développement de carrière proposées plus grandes qu'ailleurs. La répartition par site fait d'ailleurs apparaître qu'à Alençon, les hommes étaient les plus nombreux (58\%). Sur l'ensemble des salarié.e.s licencié.e.s, la catégorie professionnelle la plus représentée est celle des agents de production (41\%), mais viennent ensuite les ouvriers qualifiés (30\%), puis les techniciens $(14 \%)$, les cadres et ingénieurs constituant moins de $5 \%$ des ex-salariés. En outre, la totalité de la population se compose pour plus de la moitié de personnes sans diplôme, c'est-à-dire ayant atteint un niveau scolaire primaire et collège; cependant, près d'un tiers possède un CAP ou un BEP (30\%) tandis que $13 \%$ ont un ou des diplômes supérieurs ou au moins équivalents au niveau du baccalauréat.

Le pôle gauche du graphique, et les carrières qui le caractérisent, sont particulièrement bien illustrés par les salarié.e.s de Bayeux et de Saint-Lô, les plus petits sites. Ces deux usines rassemblent un personnel en moyenne plus jeune qu'ailleurs et plus fréquemment célibataire («Sans conjoint»). En effet, pour conserver leur emploi au sein de Moulinex, une partie des agents de production des sites ayant fermé auparavant, comme Argentan ou Granville, avait accepté un reclassement dans l'une de ces deux usines, usines qui étaient alors les plus modernes du groupe d'électroménager. La concentration dans ce secteur du plan factoriel de certains traits sociaux conduit à penser qu'une autre des difficultés rencontrées par les candidat.e.s au retour à l'emploi consiste à partager des caractéristiques personnelles et professionnelles proches. L'absence de diplôme ne leur permet pas de se distinguer des autres postulant.e.s à l'embauche sur le marché local de l'emploi. En outre, peu ont pu bénéficier de formations pour améliorer leurs compétences $(\ll<3$ formations Moulinex»). Ce parcours professionnel «horizontal» au sein de l'entreprise se caractérise par l'absence de développement de carrière et une forte rotation entre différents postes d'exécution.

Le secteur situé en bas à gauche du graphique rassemble les modalités caractéristiques d'un deuxième type d'itinéraire professionnel marqué par une ancienneté longue et un parcours en progression chez Moulinex. Ce schéma ascensionnel de «sortie de la chaîne» s'applique surtout à des agents de production parvenus à accéder à des postes d'ouvrier qualifié («Agent de production - ouvrier qualifié»). L'existence de ce profil révèle que les frontières entre les différentes catégories professionnelles 
n'étaient pas étanches chez Moulinex. Les salariés de l'usine de Falaise sont assez emblématiques de ce parcours et ont le plus souvent une conjointe également salariée dans l'entreprise. Pour une partie du personnel (les hommes plus spécifiquement), Moulinex constituait un cadre à la fois favorable à la promotion sur un marché interne du travail et propice à la construction de sa vie conjugale et familiale.

Le pôle à droite du graphique, et les carrières correspondantes, sont le mieux représentés par l'usine d'Alençon, l'une des têtes industrielles du groupe avec Cormelles-le-Royal, qui, toutes deux, rassemblaient une proportion plus importante qu'ailleurs de techniciens et agents de maîtrise (18\% contre $13 \%$ pour Bayeux et $12 \%$ à Saint-Lô) en raison de la présence de bureaux d'études et d'ateliers spécialisés. Alençon se distingue nettement des autres par un niveau moyen de diplôme légèrement plus élevé $(37 \%$ de détenteurs d'un CAP ou BEP contre un peu moins de $30 \%$ en moyenne). Ce poids des techniciens et agents de maîtrise s'explique en partie par la présence à partir de 1961 de l'école professionnelle de l'entreprise d'électroménager, qui pouvait ainsi former sur place la main-d'œuvre qualifiée dont elle avait besoin pour innover dans ses produits.

Les licencié.e.s de l'autre tête industrielle du groupe, la vaste usine de Cormelles-le-Royal dans le Calvados, possèdent des caractéristiques socioprofessionnelles proches de la moyenne de l'ensemble des ex-Moulinex, ce qui peut expliquer la position centrale de la modalité «Cormelles» sur le plan factoriel. L'usine de fours à micro-ondes située près de Caen abritait les directions industrielles du groupe, des achats et de la qualité, des ressources humaines pour la division industrie, un secteur informatique ainsi qu'un nombre légèrement plus élevé qu'ailleurs de cadres et de techniciens diplômés, jeunes et recrutés tardivement pour organiser les restructurations à partir de $1998^{(16)}$.

La projection sur le plan des modalités supplémentaires ${ }^{(17)}$ illustrant les situations retrouvées ne fait pas apparaître une division entre emploi et non-emploi mais une partition entre, d'une part, les positions de retrait ou d'instabilité vis-à-vis du marché du travail et, d'autre part, les situations durables sur celui-ci. En effet, la partie à gauche du plan factoriel correspond aux modalités de la recherche d'emploi, de l'insertion professionnelle précaire et/ou à temps partiel et des mesures

(16) Toutefois, leur très faible nombre dans notre échantillon (trente et un) explique leur position extrêmement décentrée dans la partie en haut à droite du plan factoriel.

(17) Les variables supplémentaires reflètent non seulement les situations retrouvées depuis le licenciement et la perception des effets de la perte d'emploi sur la santé mais aussi le sentiment de fierté et l'existence d'un projet de reconversion professionnelle. Elles sont notées entre parenthèses dans le plan factoriel. d'âge. À droite du plan factoriel, c'est-à-dire dans le pôle conception du travail, se situe la modalité de la réinsertion professionnelle stable. Cette partition des destins des salarié.e.s deux ans après leur licenciement se calque assez justement sur la division spatiale de l'entreprise repérée précédemment. En effet, aux catégories professionnelles les moins élevées chez Moulinex correspondent les situations les moins ancrées sur le marché du travail (chômage, emploi précaire, attentes de mesures d'âge) alors qu'aux postes les plus qualifiés et diplômés s'adjoignent les positions professionnelles les mieux assurées.

Pour les salarié.e.s ayant une faible qualification, les anciens collègues de travail se transforment alors en concurrents sur le marché de l'emploi. À l'inverse, les relations syndicales ou la formation suivie dans l'entreprise sont des facteurs qui semblent ici favorables au développement des compétences et au retour à l'emploi («>1 adhésion syndicale»). En effet, dans le cas d'un plan social où les salarié.e.s issu.e.s d'une même entreprise apparaissent comme ayant des parcours professionnels très similaires, ces attributs permettent aux candidats à l'emploi de se différencier des autres ou de bénéficier de réseaux informels de soutien.

\section{Trois carrières de licencié.e.s : du rebond difficile à la transition en douceur}

En partant de l'analyse des correspondances précédemment établies, nous dressons dans cette troisième et dernière partie une typologie compréhensive des carrières de licencié.e.s. À la différence des classifications qui visent à apporter un ordre de grandeur du nombre d'individus concernés, le but recherché ici est de typifier les expériences vécues du licenciement en les enrichissant des témoignages de la vingtaine de salarié.e.s rencontré.e.s lors d'entretiens approfondis. Les différentes carrières seront donc présentées en fonction du caractère typique de leurs caractéristiques et non du point de vue de leur représentativité statistique.

La notion de carrière sera utile ici pour articuler trajectoire objective et expérience subjective et voir la manière dont la segmentation de la main-d'œuvre dans l'espace professionnel de Moulinex se retrouve dans les trajectoires sur le marché du travail deux ans après. En intégrant l'expression des représentations et sentiments des salarié.e.s, elle révèle comment ces derniers et ces dernières composent avec les conditions et les contraintes auxquelles ils/ elles font face et comment ils/elles les interprètent. 


\section{Le rebond difficile des femmes ouvrières spécialisées}

Le premier type de carrière repéré se trouve dans le quadrant en haut à gauche du plan factoriel. Les femmes ${ }^{(18)}$ et les ouvriers non qualifiés («Agents de production»), qui ont conservé durant toute leur carrière cette position, en sont les meilleurs représentants. Le travail sur convoyeur et, plus tard, sur les unités autonomes de production, était massivement assuré par une main-d'œuvre féminine soumise à l'exigence d'accroissement des rendements et aux menaces récurrentes de suppressions de postes. Cette situation conjuguant insatisfaction professionnelle et rapport incertain à l'emploi rappelle l'intégration dite «disqualifiante» repérée par Serge PAUgam (2000). Mais la souffrance physique au travail et l'ambiance dégradée dans les ateliers n'empêchaient pourtant pas une implication forte dans l'activité de production et les collectifs de travail. La fin de Moulinex a pu représenter une épreuve mais aussi être accueillie positivement, comme une invitation à se projeter dans un emploi plus épanouissant; en outre, quitter un travail démarré souvent très tôt dans la carrière et vecteur de souffrances (que l'on retrouve à travers la modalité associée «Santé meilleure depuis le licenciement») a pu également être accueilli comme un soulagement.

Plus des trois quarts des agents de production n'ont connu d'autre expérience professionnelle que Moulinex. Pourtant, la majorité d'entre eux avait généralement une idée assez claire du nouveau métier qu'ils envisageaient d'exercer après leur licenciement. C'est le cas de madame Valmy, qui souhaite quitter le monde de l'industrie et retrouver un emploi lié à la conduite de véhicules, petits ou gros. Elle explique son tiraillement entre son désir de réorientation professionnelle et l'urgence de devoir retrouver un travail :

" Je sais que je veux reprendre mais je ne sais pas dans quel domaine. Je sais que je veux retravailler vite; ça dépend des opportunités qui se présentent. Je sais que je voudrais être moniteur d'auto-école et disons que s'il y a un poste de cariste demain, je me présente pour être cariste; je ne suis pas bornée sur un truc, j'aimerais mieux aller vers un truc qui me plaît plus. Moi, je ne veux plus retourner en usine. [...] Ils proposent dans les petites annonces [...] : "Tiens, ils cherchent une coiffeuse”, mais moi ça ne m'intéresse pas!»

(Madame Valmy, 47 ans, en couple, agent de production chez Moulinex, Falaise, sans emploi à la recherche d'un emploi.)

Mais les espoirs de reconversion dans un autre emploi sont souvent bridés par des questions d'âge, de manque de qualification ou d'expérience professionnelle rendant difficile la réalisation du projet

(18) Les trois quarts d'entre elles avaient un diplôme inférieur au CAP-BEP.
(«Projet NR»). L'élaboration d'un projet professionnel se trouve aussi contingentée par de nombreux obstacles: passage par une formation longue ou un concours d'accès, discrimination implicite à l'embauche, etc. Les conseillers à l'emploi se font d'ailleurs parfois l'écho de ces normes de discrimination afin de décourager les projets de reconversion qu'ils considéraient comme utopiques; madame Caussin en a fait la malheureuse expérience :

«Moi, je voulais faire du standard, de l'accueil [...] mais quand je suis allée voir le psychologue de l'Afpa [Association nationale pour la formation professionnelle des adultes], vous savez ce qu'il m'a dit? Qu'un patron préférera prendre une jeune de 20 ans qui sait faire des choses! ”

(Madame Caussin, 52 ans, en couple, agent de production chez Moulinex, Cormelles, sans emploi à la recherche d'un emploi.)

Un âge assez élevé mais encore intermédiaire compromet les chances de retour à l'emploi et d'accès à la formation mais ne permet pas non plus de bénéficier d'une mesure d'âge. Outre cette situation en étau, les ex-salarié.e.s de cette classe d'âge souffrent également d'être un groupe socialement et professionnellement homogène. Le licenciement intervient dans un parcours professionnel caractérisé par l'absence de diplôme, et par une expérience au sein d'une seule et même entreprise, comme dans le cas de nombreuses femmes de Moulinex. Une femme, agent de production, m'avait confié lors d'un entretien : "Sur mon CV j'ai retiré ma photo car je ressemble à une ouvrière.»

Avec le licenciement, les salarié.e.s font l'expérience d'un renversement du sens de leur intégration professionnelle. Alors que dans l'emploi, l'identité et l'expérience de travail chez Moulinex étaient considérées comme le gage d'une insertion sociale réussie - en raison notamment des avantages sociaux ${ }^{(19)}$ et de la stabilité associée à une grande entreprise renommée (RoupNEL-FuENTES, 2011) -, hors de ce cadre, elles se transforment en stigmate. Le personnel d'une grande entreprise comme Moulinex pâtit des représentations négatives qui circulent à son sujet: «salariés nantis », «travailleurs hyper syndiqués», etc. La fierté d'avoir appartenu au groupe d'électroménager se trouve alors mise en berne («Moins fier d'être Moulinex») et des participants à l'enquête confient même dissimuler à leur potentiel employeur leur identité d'ancien Moulinex.

Quand il y a réinsertion professionnelle, l'emploi est précaire, voire ultra-précaire. Madame Gallien raconte avoir trouvé un emploi de trente heures de ménage dans le mois, rémunéré par le biais de chèques emploi-service universels. À ses yeux, c'est

(19) Les salariés pouvaient bénéficier de congés supplémentaires, d'une prime d'ancienneté, d'un treizième mois et d'une prime d'équipe, de transport et de panier (la moitié du repas était prise en charge par l'employeur). 
le «dernier recours » pour éviter à tout prix l'installation prolongée dans le chômage. "Décrocher un contrat » devient une quête obsédante et entraîne souvent un stress que ravivent les rencontres avec les agents de reclassement ou du service public de l'emploi. La proximité des modalités de l'insertion professionnelle instable («Emploi à durée limitée») et du chômage («Sans emploi») montre que loin de s'opposer, ces deux situations fonctionnent en réalité de concert et s'alimentent mutuellement. Le parcours sur le marché du travail est pavé de phases plus ou moins longues de chômage, d'emplois à durée déterminée, de missions d'intérim et de stages en entreprise. En faisant l'expérience de la perte d'emploi, beaucoup de licencié.e.s font celle de la précarité professionnelle. Les petits contrats, les formations de remise à niveau et les évaluations en milieu de travail (EMT) sont vus plus souvent comme des palliatifs pour échapper au chômage que comme un marchepied à une réinsertion professionnelle assurée. Dans le cas de ces carrières, le licenciement économique a eu pour effet de rendre vulnérables des trajectoires professionnelles considérées comme assurées et de rendre «problématiques» des caractéristiques qui dans l'entreprise ne l'étaient pas.

\section{Les "sorties de chaîne" des anciens ouvriers professionnels}

Le secteur situé au sud-ouest du graphique regroupe les modalités illustrant un itinéraire marqué par une progression professionnelle chez Moulinex et un mouvement de «sortie de la chaîne» de production. Ce type de carrière se distingue de la précédente par sa forme ascensionnelle et concerne les salarié.e.s qui, d'agent de production, ont atteint la position d'ouvrier qualifié.

$\mathrm{Du}$ fait de leur carrière, beaucoup se sentent redevables à l'entreprise mais aussi à leurs efforts personnels, déployés au travers de la formation professionnelle et de leur implication dans le travail. Leur lieu de travail était aussi souvent un lieu de rencontres d'où sont nées des unions conjugales («Conjoint ex-Moulinex»).

L'histoire de madame Ledoze est représentative de ce type de trajectoire ascendante au sein de l'entreprise, même si elle est atypique au sens où ces ascensions étaient davantage conjuguées au masculin. Madame Ledoze a débuté chez Moulinex en tant qu'agent de production puis est devenue monitrice et enfin, chef d'équipe: "J'ai grandi avec l'entreprise», explique-t-elle. Sa carrière professionnelle occupait une place importante dans sa vie; en outre, elle vivait seule sans enfant. Elle explique comment son cheminement chez Moulinex a suivi le développement de l'entreprise, et valorise les compétences techniques qu'elle y a acquises.

«J'ai grandi avec l'électronique parce que je ne savais pas ce qu'était un condensateur, je ne savais pas ce qu'était un service intégré, tout ce qu'était la technologie des pièces [...] et je suis la première personne qui a démarré l'électronique à Saint-Lô. J'ai appris à lire les résistances, j'ai appris à lire les condensateurs; donc j'ai fait une formation, j'allais deux fois par semaine à Marie-Curie. »

(Madame Ledoze, 55 ans, seule, ouvrière qualifiée chez Moulinex, Saint-Lô, préretraitée.)

À la différence des ouvriers qualifiés « de métier», les représentants de ce type de carrière ont été formés en situation de travail, par la formation interne et «sur le tas», en conciliant travail et apprentissage. Mais la formation reçue en interne restait adossée aux besoins et aux évolutions de l'entreprise. S'étalant sur une période assez longue, elle portait sur un domaine d'activité spécifique alors novateur (électronique, sérigraphie, etc.). L'expérience professionnelle de ces salarié.e.s promu.e.s s'est donc construite dans des ateliers spécialisés, sur des appareils précis ou certains types de machine. La contrepartie est leur forte spécialisation dans certains champs d'activité de l'électroménager. Le transfert de compétences est donc plus difficile dans le cadre d'une réinsertion professionnelle. De plus, si ce type de carrière se distingue de la précédente par sa forme ascensionnelle, ces trajectoires de «sortie de chaîne» sont très individuelles et isolées. En effet, ses représentants avaient quitté le monde des agents de production sans jamais avoir pu réellement intégrer celui des ouvriers qualifiés.

Il existait pour eux une véritable imbrication de la vie personnelle avec la vie professionnelle. Le travail chez Moulinex étant constitutif de leur identité personnelle, la rupture avec l'emploi est une rupture avec eux-mêmes qui rappelle l'expérience du chômage total repéré par Dominique SCHNAPPER (1979). Dans le cas de ces carrières, le licenciement va causer un décrochage, une coupure dans une trajectoire professionnelle autrefois perçue comme assurée si bien que ces ex-salarié.e.s souffrent particulièrement du vide laissé par l'absence d'activité professionnelle ( Santé moins bonne depuis le licenciement»). Du fait de leur ancienneté élevée chez Moulinex, ils/elles se retrouvent plus souvent concerné.e.s par les dispositifs de mesure d'âge, comme l'a été madame Ledoze, mais ont le sentiment d'une carrière interrompue trop tôt.

\section{La poursuite de carrière des hommes jeunes et qualifiés}

Le secteur sud-est correspond aux carrières d'un personnel plutôt masculin, qualifié et syndiqué. Très souvent, la profession d'ouvrier qualifié a constitué une étape vers celle de technicien, d'agent de maîtrise, de contremaître voire d'ingénieur. Moulinex fournissait alors un cadre favorable à l'épanouissement professionnel, au développement des compétences ( $\geq 3$ formations Moulinex») et à la satisfaction dans le travail. Contrairement aux « sorties de chaîne » où les formations et compétences 
ont été obtenues plus tardivement dans la trajectoire professionnelle et entièrement à l'intérieur de l'entreprise, dans ce type de carrière, le diplôme est obtenu très tôt et les compétences entretenues tout le long du parcours professionnel dans l'entreprise. Les formations suivies au sein de Moulinex sont nombreuses, variées et le plus souvent adossées à des perspectives d'évolution professionnelle.

Ce secteur du graphique ne peut être rattaché à une situation particulière sur le marché du travail : il se situe entre les variables de l'emploi assuré et celles des mesures d'âge. Lorsque les ex-salarié.e.s sont en recherche d'emploi, ils en profitent pour suivre des formations ou nourrir des projets personnels en lien avec leur métier, les projets de reconversion professionnelle étant le plus souvent exclus. À plusieurs égards, ce type de carrière s'apparente à l'expérience du «chômage des cadres» repérée par Dominique SCHNAPPER (1979) et explorée dans les années 1990 par Sophie Pochic (2001b) qui consacrent ainsi tout le temps dégagé à leur recherche d'emploi ou à une création/reprise d'entreprise. Plutôt que comme une épreuve, cet ingénieur de l'usine de Saint-Lô dépeint cette période de deux ans comme une «expérience» humaine :

"L'expérience du chômage, c'est une expérience sur soi, on apprend de soi, on apprend aussi sur les autres. Par exemple, dans le chômage, les amitiés se révèlent...»

(Monsieur Ibert, 52 ans, en couple, ingénieur à Moulinex Saint-Lô, inscrit à l'Agence nationale pour l'emploi [ANPE].)

De statuts plus élevés au sein de la hiérarchie professionnelle de Moulinex, les représentants de ces carrières étaient aussi plus proches des instances décisionnelles et syndicales et mieux au fait des problèmes rencontrés par l'entreprise (cf. supra). $\mathrm{Au}$ moment où la survie du groupe industriel était la plus menacée, de nombreux cadres, ingénieurs ou techniciens ont anticipé la recherche d'un nouvel emploi. Plus prévisible, le licenciement a constitué une étape moins déstabilisante («Santé identique depuis le licenciement»). Les plus diplômés, les plus mobiles et les plus jeunes ont réussi à effectuer une transition en douceur, même si les cadres issus de la promotion professionnelle interne ne se sont pas obligatoirement réinsérés rapidement.

L'emploi chez Moulinex était considéré comme épanouissant et offrant des opportunités de carrière. La fierté d'avoir appartenu à l'entreprise d'électroménager $n^{\circ} 1$ en Europe est d'ailleurs restée le plus souvent intacte («Toujours fier d'être Moulinex»). Les anciennes relations de travail et l'esprit de camaraderie dans les ateliers spécialisés ou les bureaux d'étude, ont été peu entamés depuis la fin de l'emploi. Leur niveau de qualification et de spécialisation leur assure de meilleures chances de pouvoir retrouver un nouvel emploi, avec des garanties professionnelles et salariales relativement proches de celles proposées chez Moulinex. Le licenciement est le plus souvent présenté comme un passage d'un environnement professionnel à un autre, il est banalisé dans les discours des plus diplômés. La recherche d'un nouvel emploi est même parfois décrite comme une expérience stimulante: "Changer d'entreprise, c'est motivant", confiera madame Ondine, 40 ans, bachelière et qui, après avoir été longtemps intérimaire, fut embauchée chez Moulinex à Bayeux.

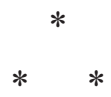

La démarche longitudinale suivie ici, consistant à relier le passé dans l'entreprise au devenir des ex-Moulinex deux ans après leur licenciement, avait pour objectif de révéler la diversité des trajectoires sur le marché du travail. Elle a permis de relativiser l'événement de la perte d'emploi, c'est-à-dire de le resituer dans le contexte professionnel et social dans lequel il est intervenu pour mieux en éclairer la portée sur un temps long. Cette mise en regard des positions dans l'entreprise et des situations retrouvées hors de celle-ci a montré que la segmentation de la main-d'œuvre au sein de Moulinex, fortement dépendante du sexe, de l'âge et de la localisation géographique, se trouve transposée dans les trajectoires des licencié.e.s deux ans après le licenciement. Si les plus âgés et anciens dans l'emploi sont sortis rapidement du marché du travail grâce aux mesures d'âge, les femmes agents de production du Calvados, confrontées aux contrats à durée limitée et au chômage, ont plutôt des itinéraires tortueux et incertains. Leurs parcours se distinguent nettement de la carrière des techniciens et agents de maîtrise : cette fraction masculine des salariés, plus qualifiée et plutôt issue de l'usine d'Alençon, se réinsère plus facilement et, généralement, de manière stable sur le marché du travail. Notre étude conforte donc les constats classiques de l'hétérogénéité des expériences vécues de la perte d'emploi, qui peut représenter une opportunité de reconversion ou signifier l'enfermement dans la galère des petits boulots.

Le net désavantage repéré pour le personnel féminin et âgé fait aussi écho à la discrimination pratiquée par les recruteurs envers les femmes seniors et ouvrières spécialisées. De même, les inégalités territoriales entre les salariés du Calvados et ceux des usines de l'Orne et de la Manche, invitent à prendre en compte à la fois le dynamisme du bassin d'emploi et le nombre de demandeurs d'emploi sur le territoire. Dans le cas de Cormelles-le-Royal, le type d'emplois disponibles aux alentours et la concentration de candidats à l'embauche ont réduit les chances de réinsertion professionnelle des salarié.e.s peu qualifié.e.s de cette usine. L'analyse en profondeur du devenir des ex-Moulinex a mis 
également en lumière l'importance, sur les trajectoires post-licenciement, du capital professionnel dont le poste occupé, l'adhésion syndicale, la formation et l'ancienneté sont les éléments constitutifs. Si la concentration du chômage et la précarité des emplois disponibles localement ont eu un effet défavorable pour la réinsertion professionnelle, les réseaux des anciens collègues, voire les camarades du syndicat, ont parfois joué le rôle d'intermédiaires et favorisé l'embauche d'ex-Moulinex dans certaines entreprises des environs.

Dans un contexte de chômage de masse et, a fortiori, dans le cadre d'un licenciement économique, le capital professionnel permet finalement de «faire la différence» et d'introduire de la variété dans des parcours professionnels issus d'une même entreprise. Au contraire, le fait de partager des caractéristiques sociales et professionnelles proches sanctionne fortement des salarié.e.s peu qualifié.e.s, faiblement formé.e.s et sans appartenance syndicale. L'absence relative d'influence du diplôme révèle qu'il ne joue pas ici le rôle de différenciateur entre des travailleurs qui, issus d'une même entreprise, souffrent $d$ '«indistinctabilité» sociale sur le marché du travail où, avec le temps, d'anciens collègues deviennent de futurs concurrents dans l'accès à l'emploi.

Ces résultats conduisent assez directement à poser la question de la sécurisation des trajectoires professionnelles. L'idée de marchés transitionnels du travail favorisant les ramifications d'une position donnée à une autre par le biais de périodes de congés, de formation ou de reconversion professionnelle, devient un enjeu essentiel. Mais le principe d'égalité entre les licencié.e.s pour motif économique que poursuit un plan social en appliquant de manière indistincte les mesures d'aide au retour à l'emploi se heurte aux fortes disparités de destins des chômeurs. La mise en lumière des inégalités dans le devenir entre salariés d'une même entreprise semble donc plaider pour un accompagnement renforcé et différencié en faveur des personnes les plus éloignées de l'emploi (à l'image des réformes actuelles du service d'accompagnement de Pôle emploi). Penser de manière non pas individuelle mais catégorielle et localisée le traitement du chômage de masse constitue une piste pour éviter l'éviction durable d'une partie de la population active du marché du travail.

\section{Bibliographie}

Amossé T., Perraudin C., Petit H. (2012), «Mobilité et segmentation du marché du travail: quel parcours professionnel après avoir perdu ou quitté son emploi ?», Économie et statistique, $\mathrm{n}^{\circ} 450$, pp. 79-105.

Benarrosh Y. (1995), «Itinéraires de chômage après un licenciement collectif dans l'industrie textile», communication aux deuxièmes journées d'étude Céreq - Lasmas-IdL, L'analyse longitudinale du marché $d u$ travail, Caen, 28 et 29 juin, Documents Série Séminaires, $\mathrm{n}^{\circ} 112$, pp. 155-189.

Bobbio M., Gratadour C., Zegnani S. (2009), «Le reclassement professionnel des salariés licenciés pour motif économique: comparaison entre le contrat de transition professionnelle, la convention de reclassement personnel et l'accompagnement classique de l'ANPE», Premières informations - Premières synthèses, $\mathrm{n}^{\circ}$ 43-3.

Canceill G., Pignoni M.-T. (2000), «Acteurs locaux de l'emploi », Travail et emploi, $\mathrm{n}^{\circ} 81$, dossier.

DARMON M. (2008), «La notion de carrière: un instrument interactionniste d'objectivation», Politix, n 82, pp. 149-167.

Demazière D. (1995), La sociologie du chômage, Paris, La Découverte.

Demazière D. (2003), «Matériaux qualitatifs et perspective longitudinale. La temporalité des parcours professionnels saisis par les entretiens biographiques », communications aux dixièmes journées d'étude Céreq
- Lasmas-IdL, Les données longitudinales dans l'analyse du marché du travail, Caen, 21, 22 et 23 mai, Documents Série Séminaires, $\mathrm{n}^{\circ} 171$, pp. 75-89.

Dif-Pradalier M., Reix F. (2012), Figures de salariés CFTC en lutte. Les cas de Continental et de Nortel, Pantin, Confédération française des travailleurs chrétiens.

Dietrich P., Loison M., Roupnel M. (2010), «Articuler les approches quantitative et qualitative», in Paugam S., L'enquête sociologique, Paris, Presses universitaires de France, pp. 207-222.

Glaser B. G., Strauss A. L. (1971), Status passage, London, Routledge and Kegan Paul.

Granovetter M. S. (1974), Getting a job: study of contacts and careers, Cambridge, Harvard university press.

Jolivet A. (2003), «Âge et relation d'emploi : les mécanismes d'une sélection défavorable aux travailleurs plus âgés », Revue d'économie politique, vol. 113, n 1 , pp. 15-35.

Larose C., avec Béroud S., Mouriaux R., Rahbi M. (2001), Cellatex. Quand l'acide a coulé, Paris, Syllepse.

Lazarsfeld P. F., Jahoda M., Zeisel H. (1981), Les chômeurs de Marienthal, Paris, Éditions de Minuit (1 ${ }^{\text {re }}$ édition 1932).

Ledrut R. (1966), Sociologie du chômage, Paris, Presses universitaires de France. 
Linhart D., avec Rist B., Durand E. (2002), Perte d'emploi, perte de soi, Ramonville-Saint-Agne, Érès.

Margolis D. N. (2002), «Licenciements collectifs et délais de reprise d'emploi», Économie et statistique, $\mathrm{n}^{\circ} 351$, pp. 65-80.

Maruani M. (2006), Travail et emploi des femmes, Paris, La Découverte.

Outin J.-L., Perrier-Cornet F. (1991), Longues durées de chômage et sites en reconversion. La diversité des trajectoires professionnelles au Creusot et à Montceaules-Mines, Dijon, Direction régionale du travail et de l'emploi.

Paugam S. (2000), Le salarié de la précarité. Les nouvelles formes de l'intégration professionnelle, Paris, Presses universitaires de France.

Pignoni M.-T., Poujouly C. (1999), «Les parcours des chômeurs récurrents. Une comparaison de typologies quantitatives et récits biographiques», communication aux sixièmes journées d'études Céreq, «Insertion, transition professionnelle et identification de processus », Clermont-Ferrand, 27 et 28 mai, Documents Série Séminaires, $\mathrm{n}^{\circ} 142$, pp. 271-288.

Pochic S. (2001a), «La menace du déclassement. Réflexions sur la genèse et l'évolution des projets professionnels de cadres au chômage », Revue de l'Ires, $\mathrm{n}^{\circ} 35$, pp. 61-88.
Pochic S. (2001b), Les cadres à l'épreuve de l'employabilité. Le chômage des cadres dans les années 1990, Thèse de sociologie, Université d'Aix-Marseille.

RoupNel-Fuentes M. (2007), Une rupture totale. Le licenciement massif des salariés de Moulinex, Thèse de sociologie, École des hautes études en sciences sociales.

Roupnel-Fuentes M. (2011), Les chômeurs de Moulinex, Paris, Presses universitaires de France.

Roupnel M. (2014a), «Les cadres sociaux du reclassement. Sur le devenir des licenciés de Moulinex », in Paugam S. (dir.), L'intégration inégale. Force, fragilité et ruptures des liens sociaux, Paris, Presses universitaires de France, pp. 313-328.

Roupnel-Fuentes M. (2014b), «Souffrances au chômage. Sur les femmes et les hommes de Moulinex», Travail, genre et sociétés, $\mathrm{n}^{\circ} 32$, pp. 99-107.

SChNAPPER D. (1994), L'épreuve du chômage, Paris, Gallimard.

SCHNAPPER D. (2005), La compréhension sociologique : démarche de l'analyse typologique, Paris, Presses universitaires de France.

Terrail J.-P. (1990), Destins ouvriers. La fin d'une classe?, Paris, Presses universitaires de France.

Trotzier C. (2006), «Le choc du licenciement : femmes et hommes dans la tourmente», Travail, genre et sociétés, $\mathrm{n}^{\circ} 16$, pp. 19-37. 
Annexe

ACM, coordonnées de l'ensemble des modalités et leurs contributions aux axes des modalités actives

\begin{tabular}{|c|c|c|c|c|}
\hline & $\begin{array}{c}\text { Coordonnées } \\
\text { Axe 1 }\end{array}$ & $\begin{array}{c}\text { Coordonnées } \\
\text { Axe } 2\end{array}$ & $\begin{array}{c}\text { Contribution } \\
\text { Axe } 1\end{array}$ & $\begin{array}{c}\text { Contribution } \\
\text { Axe } 2\end{array}$ \\
\hline$<39$ ans & 1,56 & 1,41 & 0,087 & 0,114 \\
\hline $40-49$ ans & $-0,21$ & 0,35 & 0,006 & 0,026 \\
\hline$>50$ ans & $-0,14$ & $-0,52$ & 0,003 & 0,082 \\
\hline Aucun diplôme & $-0,65$ & 0,10 & 0,088 & 0,003 \\
\hline CAP BEP & 0,59 & $-0,62$ & 0,037 & 0,065 \\
\hline BAC et plus & 1,59 & 0,97 & 0,114 & 0,068 \\
\hline 1 adhésion syndicale et plus & 0,08 & $-0,41$ & 0,000 & 0,027 \\
\hline Aucune adhésion syndicale & $-0,03$ & 0,17 & 0,000 & 0,011 \\
\hline Homme & 0,72 & $-0,37$ & 0,082 & 0,035 \\
\hline Femme & $-0,57$ & 0,30 & 0,065 & 0,028 \\
\hline Alençon & 0,17 & $-0,42$ & 0,002 & 0,026 \\
\hline Bayeux & $-0,155$ & 0,62 & 0,000 & 0,023 \\
\hline Cormelles & 0,09 & 0,02 & 0,001 & 0,000 \\
\hline Falaise & $-0,37$ & $-0,13$ & 0,005 & 0,001 \\
\hline Saint-Lô & $-0,49$ & 0,82 & 0,005 & 0,022 \\
\hline Agent de production & $-0,75$ & 0,43 & 0,085 & 0,044 \\
\hline Agent de production - ouvrier qualifié & $-0,15$ & $-0,37$ & 0,001 & 0,016 \\
\hline Ouvrier qualifié & 0,762 & $-0,78$ & 0,016 & 0,028 \\
\hline Employé, technicien, agent de maîtrise & 0,87 & $-0,34$ & 0,068 & 0,017 \\
\hline Cadre & 1,76 & 1,29 & 0,041 & 0,035 \\
\hline$\leq 20$ années à Moulinex & 1,19 & 0,93 & 0,111 & 0,111 \\
\hline 20-29 années à Moulinex & $-0,36$ & 0,06 & 0,016 & 0,000 \\
\hline$\geq 30$ années à Moulinex & $-0,31$ & $-0,55$ & 0,014 & 0,072 \\
\hline Conjoint ex-Moulinex & $-0,17$ & $-0,54$ & 0,003 & 0,049 \\
\hline Conjoint non Moulinex & 0,24 & 0,21 & 0,011 & 0,013 \\
\hline Sans conjoint & $-0,49$ & 0,28 & 0,014 & 0,007 \\
\hline $0-1-2$ formations & $-0,52$ & 0,31 & 0,051 & 0,029 \\
\hline 3 formations et plus à Moulinex & 0,59 & $-0,35$ & 0,059 & 0,033 \\
\hline Emploi à durée non limitée & 0,633 & 0,23 & & \\
\hline Emploi à durée limitée & $-0,22$ & 0,26 & & \\
\hline Sans emploi & $-0,10$ & 0,20 & & \\
\hline En attente de mesures d'âge & $-0,32$ & $-0,39$ & & \\
\hline En mesure d'âge & $-0,17$ & $-0,49$ & & \\
\hline Santé meilleure & $-0,19$ & 0,12 & & \\
\hline Santé identique & 0,20 & $-0,06$ & & \\
\hline Affecté moralement & 0,28 & $-0,18$ & & \\
\hline A un projet de reconversion & $-0,08$ & 0,04 & & \\
\hline Pas de projet de reconversion & 0,22 & 0,21 & & \\
\hline Projet non-réponse & 0,07 & $-0,09$ & & \\
\hline Jamais fier d'être à Moulinex & $-0,18$ & 0,06 & & \\
\hline Toujours fier d'être à Moulinex & 1,56 & 1,41 & & \\
\hline Moins fier d'être à Moulinex & $-0,21$ & 0,35 & & \\
\hline
\end{tabular}

